\title{
Coherent optical pulse dynamics in nanocomposite plasmonic Bragg gratings
}

\author{
Ildar R. Gabitov ${ }^{1,2}$, Alexander O. Korotkevich ${ }^{2}$, Andrei I. Maimistov ${ }^{3}$, and \\ Joseph B. McMahon ${ }^{4}$ \\ 1 Department of Mathematics, University of Arizona \\ 617 North Santa Rita Avenue, Tucson, AZ 85721, USA \\ gabitov@math.arizona.edu \\ 2 L.D. Landau Institute for Theoretical Physics, Russian Academy of Sciences \\ 2 Kosygin Street, Moscow, 119334, Russian Federation \\ kao@itp.ac.ru \\ 3 Department of Solid State Physics, Moscow Engineering Physics Institute \\ Moscow, 115409, Russian Federation \\ maimistov@pico.miphi.ru \\ 4 Program in Applied Mathematics, University of Arizona \\ 617 North Santa Rita Avenue, P.O. Box 210089, Tucson, AZ 85721-0089, USA \\ jmcmahon@math.arizona.edu
}

Summary. The propagation of solitary waves in a Bragg grating formed by an array of thin nanostructured dielectric films is considered. A system of equations of Maxwell-Duffing type and describing forward- and backward-propagating waves in such a grating, is derived. Exact solitary wave solutions are found, analyzed, and compared with the results of direct numerical simulations.

\subsection{Introduction}

The last decade has been a period of rapid progress in the field of photonic crystals [1]-[10]. In particular, the one-dimensional case of a resonant Bragg grating [1]-[5] or a resonantly absorbing Bragg reflector (RABR) [6]-[8] has been studied extensively. In the simplest case a resonant Bragg grating consists of a linear homogeneous dielectric medium containing an array of thin films with resonant atoms or molecules. The thickness of each film is much less than the wavelength of the electromagnetic wave propagating through such a structure. The interaction of ultra-short pulses and films embedded with twolevel atoms has been studied by Mantsyzov et al. [1]-[5] in the framework of the two-wave reduced Maxwell-Bloch model and by Kozhekin et al. [6]-[8]. This work demonstrated the existence of the $2 \pi$-pulse of self-induced transparency in such structures $[1,4,6]$. It was also found [8] that bright as well as dark 
solitons can exist in the prohibited spectral gap, and that bright solitons can have arbitrary pulse area.

If the density of two-level atoms is very high, then the near-dipole-dipole interaction is noticeable and should be accounted for in the mathematical model. The effect of dipole-dipole interaction on the existence of gap solitons in a resonant Bragg grating was studied in [10]; details can be found in [9]. Recent numerical simulations have yielded unusual solutions known as zoomerons. [19] The optical zoomeron was discovered and investigated recently [20] in the context of the resonant Bragg grating. A zoomeron is a localized pulse similar to an optical soliton, except that its velocity oscillates about some mean value. These works also contain careful construction of the underlying mathematical model, which is derived from first principles.

Recent advances in nanofabrication have allowed the creation of nanocomposite materials, which have the ability to sustain nonlinear plasmonic oscillations. These materials have metallic nanoparticles embedded in them [21]-[23]. In this paper we consider a dielectric material into which thin films containing metallic nanoparticles have been inserted. These thin films are spaced periodically along the length of the dielectric so that the Bragg prohibited spectral gap is centered at the plasmonic resonance frequency of the nanoparticles. We derive governing equations for slowly-varying envelopes of two counterpropagating electromagnetic waves and of the plasmonic oscillation-induced medium polarization. We find that this system of equations has the form of the two-wave Maxwell-Duffing model. We find exact solutions of this system and demonstrate that, in contrast to conventional $2 \pi$-pulses, they have nonlinear phase. We show that the stability of these solutions is sensitive to perturbation of this phase. We also study the collisions of these pulses and find that the outcomes of such collisions are highly dependent on relative phase.

\subsection{Basic Equations}

We consider a grating formed by an array of thin films which are embedded in a linear dielectric medium. In our derivation of the governing equations we follow [1]-[8], wherein Bragg resonance arises if the distance between successive films is $a=(\lambda / 2) m, m=1,2,3, \ldots$. To obtain governing equations we apply the transfer-operator approach, presented below.

\subsubsection{Transfer-operator approach}

Let us consider the ultra-short optical pulse propagation along the $X$-direction of the periodic array of thin films, which are placed at points ..., $x_{n-1}, x_{n}, x_{n+1}, \ldots$ (Fig.1). The medium between films has dielectric permittivity $\varepsilon$. Hereafter, for the sake of definiteness, we consider a TE-wave whose electric field component is parallel to the layers. All results can be generalized easily for the case of TM-polarized waves. 
It is suitable to represent the electric and magnetic strengths $\boldsymbol{E}, \boldsymbol{H}$, and the polarization of the two-level atoms ensemble $\boldsymbol{P}$ in the form of Fourier integrals

$$
\begin{gathered}
\boldsymbol{E}(x, z, t)=(2 \pi)^{-2} \int_{-\infty}^{\infty} \exp [-i \omega t+i \beta z] \boldsymbol{E}(x, \beta, \omega) d t d z \\
\boldsymbol{H}(x, z, t)=(2 \pi)^{-2} \int_{-\infty}^{\infty} \exp [-i \omega t+i \beta z] \boldsymbol{H}(x, \beta, \omega) d t d z \\
\boldsymbol{P}\left(x_{n}, z, t\right)=(2 \pi)^{-2} \int_{-\infty}^{\infty} \exp [-i \omega t+i \beta z] \boldsymbol{P}\left(x_{n}, \beta, \omega\right) d t d z
\end{gathered}
$$

Outside the films the Fourier components of the vectors $\boldsymbol{E}(x, \beta, \omega)$ and $\boldsymbol{H}(x, \beta, \omega)$ are defined by the Maxwell equations. At points $x_{n}$ these values are defined from continuity conditions. Thus, the TE-wave propagation can be described by the following system.

$$
\begin{gathered}
\frac{d^{2} E}{d x^{2}}+\left(k^{2} \varepsilon-\beta^{2}\right) E=0, \\
H_{x}=-(\beta / k) E, \quad H_{z}=-(i / k) d E / d x, \quad E_{y}=E,
\end{gathered}
$$

with boundary conditions $[11,12]$

$$
E\left(x_{n}-0\right)=E\left(x_{n}+0\right), \quad H_{z}\left(x_{n}+0\right)-H_{z}\left(x_{n}-0\right)=4 i \pi k P_{y}\left(x_{n}, \beta, \omega\right),
$$

where $k=\omega / c$. The solutions of equation (1.1) in the intervals $x_{n}<x<x_{n+1}$ can be written as

$$
\begin{aligned}
E(x, \beta, \omega) & =A_{n}(\beta, \omega) \exp \left[i q\left(x-x_{n}\right)\right]+B_{n}(\beta, \omega) \exp \left[-i q\left(x-x_{n}\right)\right], \\
H_{z}(x, \beta, \omega) & =q k^{-1}\left\{A_{n}(\beta, \omega) \exp \left[i q\left(x-x_{n}\right)\right]-B_{n}(\beta, \omega) \exp \left[-i q\left(x-x_{n}\right)\right]\right\},
\end{aligned}
$$

where $q=\sqrt{k^{2} \varepsilon-\beta^{2}}$. Hence, the amplitudes $A_{n}$ and $B_{n}$ completely determine the electromagnetic field in a RABR. Let us consider the point $x_{n}$. The electric field at $x=x_{n}-\delta(\delta<<a)$ is defined by amplitudes $A_{n}^{(L)}$ and $B_{n}^{(L)}$, and the field at $x=x_{n}+\delta$ is defined by $A_{n}^{(R)}$ and $B_{n}^{(R)}$. Continuity conditions (1.1) result in the following relations among these amplitudes

$$
\begin{gathered}
A_{n}^{(R)}+B_{n}^{(R)}=A_{n}^{(L)}+B_{n}^{(L)}, \\
A_{n}^{(R)}-B_{n}^{(R)}=A_{n}^{(L)}-B_{n}^{(L)}+4 \pi i k^{2} q^{-1} P_{S, n},
\end{gathered}
$$

where $P_{S, n}=P_{S}\left(A_{n}^{(R)}+B_{n}^{(R)}\right)$ is the surface polarization of a thin film at point $x_{n}$, which is induced by the electrical field inside the film. Thus we find 


$$
A_{n}^{(R)}=A_{n}^{(L)}+2 \pi i k^{2} q^{-1} P_{S, n}, \quad B_{n}^{(R)}=B_{n}^{(L)}-2 \pi i k^{2} q^{-1} P_{S, n} .
$$

Taking into account the strength of the electric field outside the films, we write

$$
A_{n+1}^{(L)}=A_{n}^{(R)} \exp (i q a), \quad B_{n+1}^{(L)}=B_{n}^{(R)} \exp (-i q a) .
$$

If the vectors $\psi_{n}^{(L)}=\left(A_{n}^{(L)}, B_{n}^{(L)}\right)$ and $\psi_{n}^{(R)}=\left(A_{n}^{(R)}, B_{n}^{(R)}\right)$ are introduced, then the relations (1.3) can be represented as

$$
\psi_{n}^{(R)}=\widehat{U}_{n} \psi_{n}^{(L)},
$$

where $\widehat{U}_{n}$ is the transfer operator of vector $\psi_{n}^{(L)}$ through the film located at point $x_{n}$. In the general case $\widehat{U}_{n}$ is a nonlinear operator. The relations (1.4) are represented in the vectorial form

$$
\psi_{n+1}^{(L)}=\widehat{V}_{n} \psi_{n}^{(R)},
$$

where the linear operator $\widehat{V}_{n}$ transfers the vector $\psi_{n}^{(R)}$ between adjacent thin films and is represented by the diagonal matrix

$$
\widehat{V}_{n}=\left(\begin{array}{cc}
\exp (i q a) & 0 \\
0 & \exp (-i q a)
\end{array}\right) .
$$

In this manner we define the nonlinear transfer-operator of the vector $\psi_{n}^{(L)}$ through an elementary cell of RABR:

$$
\psi_{n+1}^{(L)}=\widehat{V}_{n} \widehat{U}_{n} \psi_{n}^{(L)}=\widehat{T}_{n} \psi_{n}^{(L)} .
$$

The transfer-operator approach is frequently used in models of one-dimensional photonic crystals of linear media, e.g. distributed feedback structures [13].

In (1.5) the upper index can be omitted, and the equation can be rewritten as the following recurrence relations

$$
\begin{aligned}
& A_{n+1}=A_{n} \exp (i q a)+2 \pi i k^{2} q^{-1} P_{S, n} \exp (i q a), \\
& B_{n+1}=B_{n} \exp (-i q a)-2 \pi i k^{2} q^{-1} P_{S, n} \exp (-i q a)
\end{aligned}
$$

These recurrence relations are exact, as no approximations (e.g. slowly-varying envelope of electromagnetic pulses approximation, the long-wave approximation) have been employed. Furthermore, the surface polarization of a thin film could be calculated via different suitable models. Here we follow the works by B. Mantsyzov at al. [1]-[5], and A. Kozhekin, G. Kurizki, et al. [6]-[9], where the two-level atom model has been used.

\subsubsection{Linear response approximation}

To demonstrate that the RABR is a true gap medium, it is suitable to obtain the electromagnetic wave spectrum through a linear response approximation. In the general case we can use the following expression for polarization: 


$$
P_{S, n}=\chi(\omega)\left(A_{n}^{(R)}+B_{n}^{(R)}\right) .
$$

Substitution of this formula in (1.6),(1.7) yields

$$
\begin{aligned}
& A_{n+1}=(1+i \rho) A_{n} \exp (i q a)+i \rho B_{n} \exp (i q a), \\
& B_{n+1}=(1-i \rho) B_{n} \exp (-i q a)-i \rho A_{n} \exp (-i q a) .
\end{aligned}
$$

Here $\rho=\rho(\omega)=2 \pi k^{2} q^{-1} \chi(\omega)=2 \pi \omega c^{-1} \varepsilon^{-1 / 2} \chi(\omega)$. We employ an ansatz in which the wave is a collective motion of the electrical field in the grating. Hence

$$
A_{n}=A \exp (i k n a), \quad B_{n}=B \exp (i k n a) .
$$

Refs. (1.9),(1.10) show that the wave amplitudes $A$ and $B$ satisfy the following linear system of equations:

$$
\begin{aligned}
& A \exp (i K a)=(1+i \rho) A \exp (i q a)+i \rho B \exp (i q a), \\
& B \exp (i K a)=(1-i \rho) B \exp (-i q a)-i \rho A \exp (-i q a) .
\end{aligned}
$$

A nontrivial solution of this system exists if and only if the determinant is equal to zero, i.e.

$$
\operatorname{det}\left(\begin{array}{cc}
(1+i \rho) \exp (i q a)-\exp (i K a) & i \rho \exp (i q a) \\
-i \rho \exp (-i q a) & (1-i \rho) \exp (-i q a)-\exp (i K a)
\end{array}\right)=0
$$

If we define

$$
\begin{aligned}
& Z=\exp (i K a), \\
& G=(1+i \rho) \exp (i q a)=(\cos q a-\rho \sin q a)+i(\rho \cos q a+\sin q a),
\end{aligned}
$$

then equation (1.14) can be rewritten as the following equation in $Z$ :

$$
Z^{2}-\left(G+G^{*}\right) Z+1=0 .
$$

This equation has solutions

$$
Z_{ \pm}=\operatorname{Re} G \pm i \sqrt{1-(\operatorname{Re} G)^{2}} .
$$

If $\operatorname{Re} G \leq 1$, then $\operatorname{Re} G \pm i \sqrt{1-(\operatorname{Re} G)^{2}}=\cos K a+i \sin K a$. Hence the wave numbers $K_{ \pm}$are real-valued and satisfy the transcendental equation

$$
\cos K a=\cos q a-\rho \sin q a .
$$

If $\operatorname{Re} G>1$, then the roots of equation (1.14) are real. In this case, the wave numbers $K_{ \pm}$are pure imaginary. The condition $\operatorname{Re} G>1$ defines the frequencies of the forbidden zone. The waves with these frequencies cannot propagate in the grating. The boundaries of this forbidden zone are defined by 


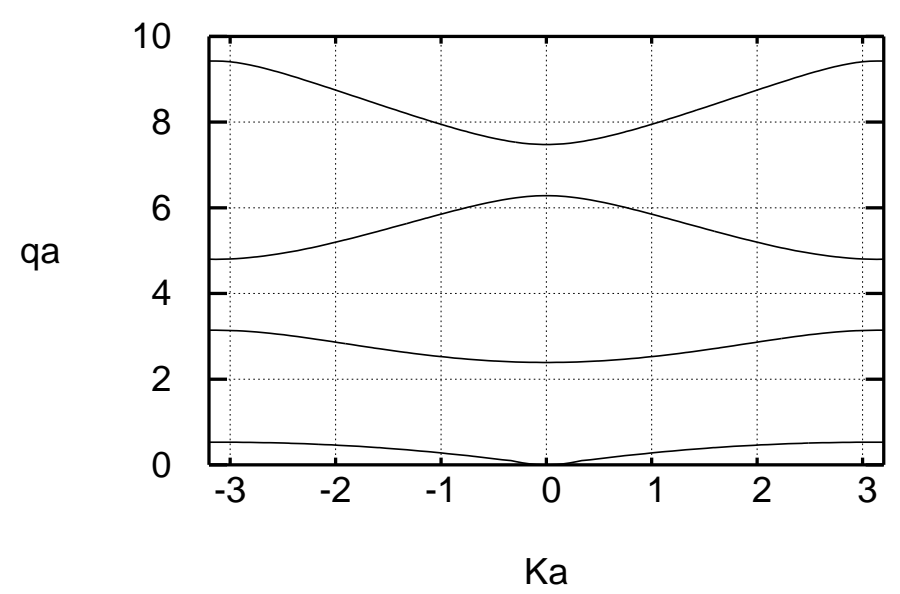

Fig. 1.1. An example of band structure, if inclusions are linear oscillators.

$$
\cos q a-\rho \sin q a=1 .
$$

The model of the resonant system containing the thin films defines the explicit form of the function $\rho=\rho(\omega)$. The form of $\rho(\omega)$ determines the dispersion relation (1.15). It should be noted that this dispersion relation ensures a series of gaps in the electromagnetic wave spectrum. Figure (1.1) represents an example of such a band structure when the inclusions are linear oscillators.

\subsubsection{Long-wave and weak nonlinearity approximations}

By using the long-wave approximation we can transform the exact equations (1.6), (1.7) into differential equations. To do so we introduce the field variables

$A(x)=\sum_{n} A_{n} \delta\left(x-x_{n}\right), \quad B(x)=\sum_{n} B_{n} \delta\left(x-x_{n}\right), \quad P(x)=\sum_{n} P_{S, n} \delta\left(x-x_{n}\right)$,

Using the integral representation for a Dirac delta-function,

$$
\delta(x)=(2 \pi)^{-1} \int_{-\infty}^{\infty} \exp (i k x) d k,
$$

we produce the following expression 


$$
\begin{aligned}
A(x) & =(2 \pi)^{-1} \sum_{n} A_{n} \int_{-\infty}^{\infty} \exp \left[i k\left(x-x_{n}\right)\right] d k \\
& =(2 \pi)^{-1} \int_{-\infty}^{\infty} \exp (i k x) \sum_{n} A_{n} \exp \left(-i k x_{n}\right) d k .
\end{aligned}
$$

It follows that the Fourier transform of $A(x)$ is then

$$
A(k)=\sum_{n} A_{n} \exp \left(-i k x_{n}\right)=\sum_{n} A_{n} \exp (-i k a n)
$$

and the Fourier transforms of $B(x)$ and $P(x)$ are

$$
B(k)=\sum_{n} B_{n} \exp (-i k a n), \quad P(k)=\sum_{n} P_{S, n} \exp (-i k a n),
$$

respectively.

The form of these spatial Fourier components ensures periodicity in $k$. For example,

$$
\begin{aligned}
A(k) & =\sum_{n} A_{n} \exp (-i k a n) \exp ( \pm 2 \pi i n) \\
& =\sum_{n} A_{n} \exp (-i k a n \pm 2 \pi i n) \\
& =\sum_{n} A_{n} \exp [-i a n(k \pm 2 \pi / a)]=A(k \pm 2 \pi / a) .
\end{aligned}
$$

¿From recurrence equations (1.6),(1.7) we have

$$
\begin{aligned}
& A(k) \exp (i k a)=A(k) \exp (i q a)+i \kappa P(k) \exp (i q a), \\
& B(k) \exp (i k a)=B(k) \exp (-i q a)-i \kappa P(k) \exp (-i q a),
\end{aligned}
$$

or

$$
\begin{aligned}
& {[\exp \{i a(k-q)\}-1] A(k)=i \kappa P(k),} \\
& {[\exp \{i a(k+q)\}-1] B(k)=-i \kappa P(k) .}
\end{aligned}
$$

The linear approximation $P(k)=\chi(\omega)[A(k)+B(k)]$ for polarization again leads us to the linear dispersion law (1.14). The system of equations (1.18),(1.19) is equivalent to the discrete equations (1.6),(1.7). At this point we have made no approximations other than the assumption on the thin films' width.

If the thin film array were absent, then the dispersion relation would be

$$
\cos k a=\cos q a .
$$


In this case the wave with amplitude $A(k)$ has $k=q$, indicating propagation to the right, while the wave with amplitude $B(k)$ has $k=-q$, indicating propagation in the opposite direction. Let us suppose that the polarization of the thin film array produces little change in wave vectors, i.e., for the rightpropagating wave the wave vector is $k=q+\delta k$, and for the opposite wave the wave vector is $k=-q+\delta k$. If we set the value of $q$ near one of the Bragg resonances, say $q=2 \pi / a+\delta q$, where $\delta q \ll 2 \pi / a$, then equations (1.6), (1.7) take the form

$$
\begin{aligned}
{[\exp (i a \delta k)-1] A(q+\delta k) } & =i \kappa P(q+\delta k), \\
{[\exp (i a \delta k)-1] B(-q+\delta k) } & =-i \kappa P(-q+\delta k) .
\end{aligned}
$$

By virtue of the periodicity conditions (1.17) these equations can be re-written as

$$
\begin{aligned}
{[\exp (i a \delta k)-1] A(\delta q+\delta k) } & =i \kappa P(\delta q+\delta k), \\
{[\exp (i a \delta k)-1] B(-\delta q+\delta k) } & =-i \kappa P(-\delta q+\delta k) .
\end{aligned}
$$

After the change of variables $\delta k=\delta \tilde{k} \pm \delta q$ we have

$$
\begin{aligned}
& {[\exp \{i a(\delta \tilde{k}-\delta q)\}-1] A(\delta \tilde{k})=i \kappa P(\delta \tilde{k})} \\
& {[\exp \{i a(\delta \tilde{k}+\delta q)\}-1] B(\delta \tilde{k})=-i \kappa P(\delta \tilde{k})}
\end{aligned}
$$

The long-wave approximation means that nonzero values of the spatial Fourier amplitudes are located near zero value of argument. Suppose that $a \delta \tilde{k}$ is small enough that $e^{i a(\delta \tilde{k}+\delta q)} \approx 1+i a(\delta \tilde{k}+\delta q)$. Then we replace equations (1.20), (1.21) with the approximate equations

$$
\begin{gathered}
i a(\delta \tilde{k}-\delta q) A(\delta \tilde{k})=i \kappa P(\delta \tilde{k}), \\
i a(\delta \tilde{k}+\delta q) B(\delta \tilde{k})=-i \kappa P(\delta \tilde{k}) .
\end{gathered}
$$

If now we return to the spatial variable, equations (1.22), (1.23) lead us to the equations of coupled-wave theory

$$
\begin{aligned}
& \frac{\partial A}{\partial x}=i \delta q A(x)+i \kappa a^{-1} P(x), \\
& \frac{\partial B}{\partial x}=-i \delta q B(x)-i \kappa a^{-1} P(x) .
\end{aligned}
$$

In these equations the fields $A(x), B(x), P(x)$ and the parameters $\delta q, \kappa$ are functions of frequency $\omega$. To obtain the final system of equations in the spatial and time variables, we perform an inverse Fourier transformation. We now assume that the envelopes of the electromagnetic waves vary slowly in time [14]. This approximation simplifies the system of coupled wave equations under consideration. 


\subsubsection{Slowly-varying envelope approximation}

In the slowly-varying envelope approximation, we assume that the approximated fields are inverse Fourier transforms of narrow wave packets, i.e. they are quasiharmonic waves [14]. For example, the quasiharmonic wave form of the electric field is

$$
E(x, t)=\mathcal{E}(x, t) \exp \left[-i \omega_{0} t\right]
$$

where $\omega_{0}$ is carrier wave frequency. The electric field $E(x, t)$ and the Fourier components of the envelope $\mathcal{E}(x, t)$ of the pulse are related by

$$
\begin{aligned}
E(x, t) & =(2 \pi)^{-1} \int_{-\infty}^{\infty} \widetilde{E}(x, \omega) \exp (-i \omega t) d \omega \\
& =(2 \pi)^{-1} \int_{-\infty}^{\infty} \widetilde{\mathcal{E}}(x, \omega) \exp \left[-i\left(\omega+\omega_{0}\right) t\right] d \omega \\
& =(2 \pi)^{-1} \int_{-\infty}^{\infty} \widetilde{\mathcal{E}}\left(x, \omega-\omega_{0}\right) \exp (-i \omega t) d \omega
\end{aligned}
$$

where the function $\widetilde{E}(x, \omega)$ is nonzero if $\omega \in\left(\omega_{0}-\Delta \omega, \omega_{0}+\Delta \omega\right)$, with $\Delta \omega \ll$ $\omega_{0}$. Hence, $\widetilde{E}\left(x, \omega+\omega_{0}\right)=\widetilde{\mathcal{E}}(x, \omega)$. Thus, if we have some relation for $\widetilde{E}(x, \omega)$, then the analogous relation for $\widetilde{\mathcal{E}}(x, \omega)$ can be found by shifting $\omega \mapsto \omega_{0}+\omega$ in all functions of $\omega$.

Let

$A(x, t)=\mathcal{A}(x, t) \exp \left(-i \omega_{0} t\right), \quad B(x, t)=\mathcal{B}(x, t) \exp \left(-i \omega_{0} t\right), \quad P(x, t)=\mathcal{P}(x, t) \exp \left(-i \omega_{0} t\right)$.

¿From Eqs. (1.24), (1.25) it follows that the Fourier components $\mathcal{A}(x, \omega), \mathcal{B}(x, \omega)$, and $\mathcal{P}(x, \omega)$ satisfy

$$
\begin{aligned}
& \frac{\partial \mathcal{A}}{\partial x}(x, \omega)=i \delta q\left(\omega_{0}+\omega\right) \mathcal{A}(x, \omega)+i \kappa\left(\omega_{0}+\omega\right) a^{-1} \mathcal{P}(x, \omega) \\
& \frac{\partial \mathcal{B}}{\partial x}(x, \omega)=-i \delta q\left(\omega_{0}+\omega\right) \mathcal{B}(x, \omega)-i \kappa\left(\omega_{0}+\omega\right) a^{-1} \mathcal{P}(x, \omega)
\end{aligned}
$$

Since $\mathcal{A}, \mathcal{B}$ and $\mathcal{P}$ are nonzero for $\omega \ll \omega_{0}$, one can use the expansions:

$$
\delta q\left(\omega_{0}+\omega\right) \approx q_{0}-2 \pi / a+q_{1} \omega+q_{2} \omega^{2} / 2, \quad \kappa\left(\omega_{0}+\omega\right) a^{-1} \approx K_{0},
$$

where $q_{n}=d^{n} q / d \omega^{n}$ at $\omega=\omega_{0}$. In particular, $q_{1}^{-1}=v_{g}$ is the group velocity, and $q_{2}$ takes into account the group-velocity dispersion.

Considering expansions (1.28), we have the following description of the evolution of slowly-varying envelopes. 


$$
\begin{gathered}
i\left(\frac{\partial}{\partial x}+\frac{1}{v_{g}} \frac{\partial}{\partial t}\right) \mathcal{A}-\frac{q_{2}}{2} \frac{\partial^{2}}{\partial t^{2}} \mathcal{A}+\Delta q_{0} \mathcal{A}=-K_{0} \mathcal{P}, \\
i\left(\frac{\partial}{\partial x}-\frac{1}{v_{g}} \frac{\partial}{\partial t}\right) \mathcal{B}+\frac{q_{2}}{2} \frac{\partial^{2}}{\partial t^{2}} \mathcal{B}-\Delta q_{0} \mathcal{B}=+K_{0} \mathcal{P},
\end{gathered}
$$

where $\Delta q_{0}=q_{0}-2 \pi / a$. The next step requires a choice of the model for the thin films' medium. Possibilities include anharmonic oscillators, two- or three-level atoms, excitons of molecular chains, nano-particles, quantum dots, and more. First we consider the two-level atom model.

\subsubsection{Example: thin films containing two-level atoms}

Here we employ the approach developed above to derive an already known system of equations [1]. The model assumes that each thin film contains twolevel atoms. The state of a two-level atom is described by a density matrix $\hat{\rho}$. The matrix element $\rho_{12}$ describes the transition between the ground state $|2\rangle$ and excited state $|1\rangle . \rho_{22}$ and $\rho_{11}$ represent the populations of theses states. Evolution of the two-level atom is governed by the Bloch equations [15].

$$
\begin{aligned}
i \hbar \frac{\partial}{\partial t} \rho_{12} & =\hbar \Delta \omega \rho_{12}-d_{12}\left(\rho_{22}-\rho_{11}\right) A_{i n}, \\
i \hbar \frac{\partial}{\partial t}\left(\rho_{22}-\rho_{11}\right) & =2\left(d_{12} \rho_{21} A_{i n}-d_{21} \rho_{12} A_{i n}^{*}\right) .
\end{aligned}
$$

In these equations $A_{i n}$ is the electric field interacting with a two-level atom. In the problem under consideration, $A_{\text {in }}=\mathcal{A}+\mathcal{B}$, and

$$
K_{0} \mathcal{P}=\frac{2 \pi \omega_{0} n_{a t} d_{12}}{c n\left(\omega_{0}\right)}\left\langle\rho_{12}\right\rangle .
$$

Here the cornerstone brackets denote summation over all atoms within a frequency detuning of $\Delta \omega$ from the center of the inhomogeneity broadening line, $n\left(\omega_{0}\right)$ is the refractive index of the medium containing the array of thin films, and $n_{a t}$ is the effective density of the resonant atoms in the films. $n_{a t}$ is defined by $n_{a t}=N_{a t}\left(\ell_{f} / a\right)$, where $N_{a t}$ is the bulk density of atoms, $\ell_{f}$ is the film width, and $a$ is the lattice spacing.

We suppose the group-velocity dispersion is of no importance. The resulting equations are the two-wave reduced Maxwell-Bloch equations. We introduce the normalized variables

$$
e_{1}=t_{0} d_{12} \mathcal{A} / \hbar, \quad e_{2}=t_{0} d_{12} \mathcal{B} / \hbar, \quad x=\zeta v_{g} t_{0}, \quad \tau=t / t_{0} .
$$

The normalized two-wave reduced Maxwell-Bloch equations take the following form:

$$
i\left(\frac{\partial}{\partial \zeta}+\frac{\partial}{\partial \tau}\right) e_{1}+\delta e_{1}=-\gamma\left\langle\rho_{12}\right\rangle
$$




$$
\begin{aligned}
i\left(\frac{\partial}{\partial \zeta}-\frac{\partial}{\partial \tau}\right) e_{2}-\delta e_{2} & =+\gamma\left\langle\rho_{12}\right\rangle \\
i \frac{\partial}{\partial \tau} \rho_{12} & =\Delta \rho_{12}-n e_{i n} \\
\frac{\partial}{\partial \tau} n & =-4 \operatorname{Im}\left(\rho_{12} e_{i n}^{*}\right),
\end{aligned}
$$

where $\gamma=t_{0} v_{g} / L_{a}, \delta=t_{0} v_{g} \Delta q_{0}, L_{a}=\left(c n\left(\omega_{0}\right) \hbar\right) /\left(2 \pi \omega_{0} t_{0} n_{a t}\left|d_{12}\right|^{2}\right)$ is the resonant absorption length, and $\Delta=\Delta \omega t_{0}$ is the normalized frequency detuning.

We define $n=\rho_{22}-\rho_{11}, e_{i n}=e_{1}+e_{2}$ and introduce yet another change of variables:

$$
e_{i n}=e_{1}+e_{2}=f_{s} \exp (i \delta \tau), \quad e_{1}-e_{2}=f_{a} \exp (i \delta \tau), \quad \rho_{12}=r \exp (i \delta \tau) .
$$

The system of equations (1.33) - (1.36) can be rewritten as

$$
\begin{aligned}
\frac{\partial f_{s}}{\partial \zeta}+\frac{\partial f_{a}}{\partial \tau} & =0 \\
\frac{\partial f_{a}}{\partial \zeta}+\frac{\partial f_{s}}{\partial \tau} & =2 i \gamma\langle r\rangle \\
i \frac{\partial}{\partial \tau} r & =(\Delta+\delta) r-n f_{s}, \\
\frac{\partial}{\partial \tau} n & =-4 \operatorname{Im}\left(r f_{s}^{*}\right) .
\end{aligned}
$$

¿From (1.37) it follows that

$$
\frac{\partial f_{a}}{\partial \zeta}=-\frac{\partial f_{s}}{\partial \tau},
$$

which allows to rewrite (1.37)-(1.40) in the form

$$
\begin{aligned}
\frac{\partial^{2} f_{s}}{\partial \zeta^{2}}-\frac{\partial^{2} f_{s}}{\partial \tau^{2}} & =-2 i \gamma\left\langle\frac{\partial r}{\partial \tau}\right\rangle, \\
i \frac{\partial}{\partial \tau} r & =(\Delta+\delta) r-n f_{s}, \\
\frac{\partial}{\partial \tau} n & =-4 \operatorname{Im}\left(r f_{s}^{*}\right) .
\end{aligned}
$$

If we assume that the inhomogeneous broadening is absent, i.e. if the hypothesis of a sharp atomic resonant transition is true, then $\delta+\Delta=0$, and this system reduces to the Sine-Gordon equation [1]. Reference [4] presents the steady-state solution of (1.41)-(1.43) with inhomogeneous broadening taken into account. 


\subsubsection{Thin films containing metallic nanoparticles}

It was shown above that counter-propagating electric field waves $\mathcal{A}$ and $\mathcal{B}$ in the slowly-varying envelope approximation satisfy the following system of equations:

$$
\begin{gathered}
i\left(\frac{\partial}{\partial x}+\frac{1}{v_{g}} \frac{\partial}{\partial t}\right) \mathcal{A}-\frac{q_{2}}{2} \frac{\partial^{2}}{\partial t^{2}} \mathcal{A}+\Delta q_{0} \mathcal{A}=-\frac{2 \pi \omega_{0}}{c \sqrt{\varepsilon}}\langle\mathcal{P}\rangle, \\
i\left(\frac{\partial}{\partial x}-\frac{1}{v_{g}} \frac{\partial}{\partial t}\right) \mathcal{B}+\frac{q_{2}}{2} \frac{\partial^{2}}{\partial t^{2}} \mathcal{B}-\Delta q_{0} \mathcal{B}=+\frac{2 \pi \omega_{0}}{c \sqrt{\varepsilon}}\langle\mathcal{P}\rangle,
\end{gathered}
$$

where $\Delta q_{0}=q_{0}-2 \pi / a$ is the mismatch between the carrier wavenumber and the Bragg resonant wavenumber. To describe the evolution of material polarization in the slowly-varying amplitude approximation, we must model the thin films' response to an external light field. Previous work has considered various mechanisms as sources of the dielectric properties of metamaterials. In the simplest case, dielectric properties can be attributed to plasmonic oscillations, which are modeled by Lorentz oscillators. Magnetic properties can be described by the equations of a system of LC-circuits [27, 28, 29, 30, 31]. The simplest generalizations of this model include anharmonicity of plasmonic oscillations $[16,32]$ or the addition of a nonlinear capacitor into each LCcircuit [33]. In this paper we consider an array of non-magnetic thin films containing metallic nanoparticles, which have cubic nonlinear response to external fields $[21,22,16]$.

The macroscopic polarization $P$ is governed by the equation

$$
\frac{\partial^{2} P}{\partial t^{2}}+\omega_{d}^{2} P+\Gamma_{a} \frac{\partial P}{\partial t}+\kappa P^{3}=\frac{\omega_{p}^{2}}{4 \pi} E,
$$

where $\omega_{p}$ is plasma frequency and $\omega_{d}$ is dimension quantization frequency for nanoparticles. Losses of the plasmonic oscillations are taken into account by the parameter $\Gamma_{a}$. It is assumed that the duration of the electromagnetic pulse is small enough that dissipation effects can be neglected. If the anharmonic parameter $\kappa$ is equal to zero, then we have the famous Lorentz model for describing electromagnetic wave propagation and refraction in metamaterials [35, 36, 27, 28, 29, 30, 31].

Starting from the slowly-varying envelope approximation, standard manipulation leads to

$$
i \frac{\partial \mathcal{P}}{\partial t}+\left(\omega_{d}-\omega_{0}\right) \mathcal{P}+\frac{3 \kappa}{2 \omega_{0}}|\mathcal{P}|^{2} \mathcal{P}=-\frac{\omega_{p}^{2}}{8 \pi \omega_{0}} \mathcal{E}_{\text {int }}(x, t) .
$$

Terms varying rapidly in time, which are proportional to $\exp \left( \pm 3 i \omega_{0} t\right)$, are neglected. In this equation $\mathcal{E}_{i n t}$ is the electric field interacting with metallic nanoparticles. In the problem under consideration we have $\mathcal{E}_{\text {int }}=\mathcal{A}+\mathcal{B}$.

Due to the limitations of nanofabrication, the sizes and shapes of nanoparticles are not uniform. In practice, deviation from a perfectly spherical shape 
has a much larger impact on a nanoparticle's resonance frequency than does variation in diameter. This causes a broadening of the resonance line. The broadened spectrum is characterized by a probability density function $g(\Delta \omega)$ of deviations $\Delta \omega$ from some mean value $\omega_{\text {res }}$. When computing the total polarization, all resonance frequencies must be taken into account.

The contributions of the various resonance frequencies are weighted according to the probability density function $g(\Delta \omega)$; the weighted average is denoted by $\langle\mathcal{P}\rangle$ in equations (1.44),(1.45). In what follows, $n\left(\omega_{0}\right)$ denotes the refractive index of the medium containing the array of thin films, and $n_{n p}$ is the effective density of the resonant nanoparticles in films. As in the model of films containing two-level atoms, the effective density is equal to $n_{n p}=N_{n p}\left(\ell_{f} / a\right)$, where $N_{n p}$ is the bulk density of nanoparticles, $\ell_{f}$ is the width of a film, and $a$ is the lattice spacing.

We study a medium-light interaction in which resonance is the dominant phenomenon. As such, the length of the sample is smaller than the characteristic dispersion length. In this case the temporal second derivative terms in equations $(1.44,1.45)$ can be omitted. The resulting equations are the twowave Maxwell-Duffing equations. They can be rewritten in dimensionless form using the following rescaling:

$$
\begin{aligned}
e_{1} & =\mathcal{A} / A_{0}, \\
e_{2} & =\mathcal{B} / A_{0}, \\
p & =\left(4 \pi \omega_{0} /\left[\sqrt{\varepsilon} \omega_{p} A_{0}\right]\right) \mathcal{P}, \\
\zeta & =\left(\omega_{p} / 2 c\right) x, \\
\tau & =t / t_{0} .
\end{aligned}
$$

Here $t_{0}=2 \sqrt{\varepsilon} / \omega_{p}$, while $A_{0}$ is a characteristic amplitude of counterpropagating fields. In dimensionless form, the two-wave Maxwell-Duffing equations read

$$
\begin{aligned}
i\left(\frac{\partial}{\partial \zeta}+\frac{\partial}{\partial \tau}\right) e_{1}+\delta e_{1} & =-\langle p\rangle \\
i\left(\frac{\partial}{\partial \zeta}-\frac{\partial}{\partial \tau}\right) e_{2}-\delta e_{2} & =+\langle p\rangle \\
i \frac{\partial p}{\partial \tau}+\Delta p+\mu|p|^{2} p & =-\left(e_{1}+e_{2}\right),
\end{aligned}
$$

where $\mu=\left(3 \kappa \sqrt{\varepsilon} / \omega_{0} \omega_{p}\right)\left(\sqrt{\varepsilon} \omega_{p} / 4 \pi \omega_{0}\right)^{2} A_{0}^{2}$ is a dimensionless coefficient of anharmonicity, $\delta=2 \Delta q_{0}\left(c / \omega_{p}\right)$ is the dimensionless mismatch coefficient, $\Delta=2 \sqrt{\varepsilon}\left(\omega_{d}-\omega_{0}\right) / \omega_{p}$ is the dimensionless detuning of a nanoparticle's resonance frequency from the field's carrier frequency.

In a coordinate system rotating with angular frequency $\delta$,

$$
e_{1}=f_{1} e^{i \delta \tau}, \quad e_{2}=f_{2} e^{i \delta \tau}, p=q e^{i \delta \tau},
$$

equations (1.47) become 


$$
\begin{aligned}
i\left(\frac{\partial}{\partial \zeta}+\frac{\partial}{\partial \tau}\right) f_{1} & =-\langle q\rangle, \\
i\left(\frac{\partial}{\partial \zeta}-\frac{\partial}{\partial \tau}\right) f_{2} & =+\langle q\rangle, \\
i \frac{\partial q}{\partial \tau}+(\Delta-\delta) q+\mu|q|^{2} q & =-\left(f_{1}+f_{2}\right) .
\end{aligned}
$$

Further simplification of the system (1.48) can be achieved by introducing new variables

$$
f_{s}=-\left(f_{1}+f_{2}\right), \quad f_{a}=f_{1}-f_{2},
$$

which allow decoupling of one equation from the system of three equations. In these new variables the polarization $q$ is coupled with only one field variable. Simple transformations give

$$
\begin{aligned}
\frac{\partial^{2} f_{a}}{\partial \zeta^{2}}-\frac{\partial^{2} f_{a}}{\partial \tau^{2}} & =2 i \frac{\partial}{\partial \zeta}\langle q\rangle, \\
\frac{\partial^{2} f_{s}}{\partial \zeta^{2}}-\frac{\partial^{2} f_{s}}{\partial \tau^{2}} & =2 i \frac{\partial}{\partial \tau}\langle q\rangle, \\
i \frac{\partial q}{\partial \tau}+(\Delta-\delta) q+\mu|q|^{2} q & =f_{s} .
\end{aligned}
$$

As one can see, we have a coupled system of equations for $f_{s}$ and $q$.

\subsection{Solitary Wave Solutions}

We consider localized solitary wave solutions of (1.49) in the limit of narrow spectral line $\Delta \omega_{g} / \Delta \omega_{s} \ll 1$, where $\Delta \omega_{s}$ and $\Delta \omega_{g}$ are spectral widths of a signal and spectral line $g(\Delta \omega)$. In this case the spectral line can be represented as Dirac $\delta$-function: $g(\Delta \omega)=\delta(\Delta \omega)$. Equations $(1.49),(1.51)$ can then be rewritten as follows:

$$
\begin{aligned}
\frac{\partial^{2} f_{s}}{\partial \zeta^{2}}-\frac{\partial^{2} f_{s}}{\partial \tau^{2}} & =2 i \frac{\partial q}{\partial \tau} \\
i \frac{\partial q}{\partial \tau}+(\Delta-\delta) q+\mu|q|^{2} q & =f_{s}
\end{aligned}
$$

Scaling analysis of this system shows that solitary wave solutions can be represented as

$$
\begin{aligned}
f_{s} & =f_{0} F_{\Omega}(\eta)=\frac{1}{\sqrt{\mu}}\left(\frac{2 v^{2}}{1-v^{2}}\right)^{3 / 4} F_{\Omega}(\eta), \\
q & =q_{0} Q_{\Omega}(\eta)=\frac{1}{\sqrt{\mu}}\left(\frac{2 v^{2}}{1-v^{2}}\right)^{1 / 4} Q_{\Omega}(\eta), \\
\eta & =(\zeta-v \tau) \sqrt{\frac{2}{1-v^{2}}} .
\end{aligned}
$$


Here $v$ is velocity of the solitary wave, $\eta$ is a scale-invariant parameter in a coordinate system moving with the solitary wave, and functions $F_{\Omega}, Q_{\Omega}$ satisfy the following system of equations:

$$
\begin{aligned}
F^{\prime \prime} & =-i Q^{\prime}, \\
-i Q^{\prime}+\Omega Q+|Q|^{2} Q & =F .
\end{aligned}
$$

The only dimensionless parameter which remains in the system is

$$
\Omega=(\Delta-\delta) \sqrt{\frac{1-v^{2}}{2 v^{2}}},
$$

which characterizes the deviation of carrier frequency from the plasmonic frequency $\omega_{p}$ and the Bragg resonance frequency $\omega_{B r}$.

The first equation implies that $F^{\prime}=-i Q+$ constant. We seek a solitarywave solution, so we assume that $F, Q$, and their derivatives decay to zero as $|\eta| \rightarrow \infty$. Hence the constant is zero, and

$$
\begin{aligned}
i F^{\prime} & =Q \\
-i Q^{\prime}+\Omega Q+|Q|^{2} Q & =F .
\end{aligned}
$$

The system of ordinary differential equations (1.58) has integral of motion

$$
|Q|^{2}-|F|^{2}=\text { constant }
$$

For solutions decaying as $|\eta| \rightarrow \infty$, the constant is equal to zero, and

$$
|Q|^{2}=|F|^{2} .
$$

This allows the following parametrization of solutions:

$$
F(\eta)=R(\eta) e^{i \phi(\eta)}, \quad Q(\eta)=R(\eta) e^{i \psi(\eta)},
$$

where $R, \phi$, and $\psi$ are real-valued functions satisfying

$$
\begin{aligned}
R^{\prime} & =-R \sin (\phi-\psi), \\
\phi^{\prime} & =-\cos (\phi-\psi), \\
\psi^{\prime}+\Omega+R^{2} & =\cos (\phi-\psi) .
\end{aligned}
$$

If we set $\Phi=\phi-\psi$, then we have

$$
\begin{aligned}
\Phi^{\prime}-\Omega-R^{2} & =-2 \cos \Phi, \\
R^{\prime} & =-R \sin \Phi .
\end{aligned}
$$

Taking into account second equation of (1.60), the first equation can be rewritten as

$$
R \frac{d}{d R}(\cos \Phi)-\Omega-R^{2}=-2 \cos \Phi .
$$


If we set $y=\cos \Phi$, then we have

$$
R \frac{d y}{d R}+2 y=R^{2}+\Omega .
$$

The solutions of equation (1.61) have the form

$$
y=\frac{1}{4} R^{2}+\frac{\Omega}{2}+c R^{-2},
$$

where $c$ is free. Since $y=\cos \Phi$, the right-hand side must remain between -1 and 1 . As we expect $R \rightarrow 0$ as $|\eta| \rightarrow \infty, c$ must be zero. We have the conservation law

$$
\cos \Phi=\frac{1}{4} R^{2}+\frac{\Omega}{2} .
$$

Substituting (1.62) into the second equation of (1.60) and subsequent integration gives the following expression for $R$ :

$$
R^{2}=\frac{2\left(4-\Omega^{2}\right)}{\Omega+2 \cosh \left\{\sqrt{4-\Omega^{2}}\left(\eta-\eta_{0}\right)+\frac{1}{2} \ln \left(\frac{16}{4-\Omega^{2}}\right)\right\}} .
$$

The right-hand side is positive real-valued for all $\eta$ if and only if $-2<\Omega<2$. $\sqrt{4-\Omega^{2}}$ appears so often in what follows that we set $\beta=\sqrt{4-\Omega^{2}} . R^{2}$ then has the form

$$
R^{2}=\frac{2 \beta^{2}}{\Omega+2 \cosh \left\{\beta\left(\eta-\eta^{\prime}\right)\right\}},
$$

where we have combined arbitrary constant $\eta_{0}$ and the logarithm into a single constant $\left(\eta^{\prime}\right)$ in the argument of the hyperbolic cosine. Using the conservation law (1.62) we obtain an expression for $\Phi$ :

$$
\Phi=2 \arctan \left(\frac{2-\Omega}{\beta} \tanh \left\{\frac{1}{2} \beta\left(\eta-\eta^{\prime}\right)\right\}\right) .
$$

Now we integrate $\phi^{\prime}=-\cos \Phi$ and find

$$
\phi=-\frac{\Omega}{2}\left(\eta-\eta^{\prime}\right)-\arctan \left(\frac{2-\Omega}{\beta} \tanh \left\{\frac{1}{2} \beta\left(\eta-\eta^{\prime}\right)\right\}\right) .
$$

Finally, we determine $\psi$ :

$$
\begin{aligned}
\psi & =\phi-\Phi \\
& =-\frac{\Omega}{2}\left(\eta-\eta^{\prime}\right)-3 \arctan \left(\frac{2-\Omega}{\beta} \tanh \left\{\frac{1}{2} \beta\left(\eta-\eta^{\prime}\right)\right\}\right) .
\end{aligned}
$$

This pulse exists only if value of the parameter $\Omega$ are inside the interval $-2<\Omega<2$. The maximal value of the amplitude of this solitary solution is

$$
A=\sqrt{2(2-\Omega)} .
$$


The phases $\phi$ and $\psi$ are nonlinear. Their behavior is asymptotically linear as $\eta \rightarrow \pm \infty$. If $\Omega=0$, then the limiting values of the phases satisfy

$$
\begin{aligned}
|\phi(\infty)-\phi(-\infty)| & =\pi / 2 \\
|\psi(\infty)-\psi(-\infty)| & =3 \pi / 2
\end{aligned}
$$

\subsection{Energy Partition}

The total energy of the solitary wave is distributed among co/contr-propagating fields and medium polarization. Here we study energy partition between all these components. Using equations (1.49), (1.50) and conditions as $|\eta| \rightarrow \infty$, one can show that

$$
f_{a}(\eta)=-\frac{1}{v} f_{s}(\eta)=-\frac{f_{0}}{v} F(\eta)
$$

We are interested in the energies of the dimensionless fields $f_{1}, f_{2}$, and polarization $q$.

$$
\begin{aligned}
f_{1} & =\frac{1}{2}\left(f_{a}-f_{s}\right)=-\frac{f_{0}}{2}\left(\frac{1}{v}+1\right) F \\
f_{2} & =-\frac{1}{2}\left(f_{s}+f_{a}\right)=\frac{f_{0}}{2}\left(\frac{1}{v}-1\right) F \\
q & =q_{0} Q
\end{aligned}
$$

To find energies of forward- and backward-propagating waves we need to calculate an energy of a solitary wave:

$$
\begin{aligned}
E_{R}= & \int_{-\infty}^{+\infty}|F|^{2}(\eta) d \eta=\int_{-\infty}^{\infty}|Q|^{2}(\eta) d \eta= \\
\int_{-\infty}^{+\infty} R^{2}(\eta) d \eta & =8 \arctan \sqrt{\frac{2-\Omega}{2+\Omega}} .
\end{aligned}
$$

Finally we have the energies

$$
\begin{aligned}
E_{f_{1}} & =\frac{f_{0}^{2}}{4}\left(\frac{1}{v}+1\right)^{2} E_{R} \\
E_{f_{2}} & =\frac{f_{0}^{2}}{4}\left(\frac{1}{v}-1\right)^{2} E_{R} \\
E_{q} & =q_{0}^{2} E_{R} .
\end{aligned}
$$

Ratios of energies in different fields as well as polarization have the following form 


$$
\begin{aligned}
& \frac{E_{f_{1}}}{E_{f_{2}}}=\left(\frac{1+v}{1-v}\right)^{2}, \\
& \frac{E_{f_{1}}}{E_{q}}=\frac{1}{2} \frac{(1+v)}{(1-v)}, \\
& \frac{E_{f_{2}}}{E_{q}}=\frac{1}{2} \frac{(1-v)}{(1+v)} .
\end{aligned}
$$

Therefore, energy partitioning is determined by only one parameter $v$ which is dimensionless combination of main system parameters.

\subsection{Numerical simulation}

The shape and phase of the incident pulse are controllable in real experimental situation. To model pulse dynamics in the Bragg grating it is natural to consider asymptotic mixed initial-boundary value problem for equations (1.48). We define initial conditione as

$$
q(\zeta, \tau) \rightarrow 0, \quad f_{1}(\zeta, \tau) \rightarrow 0, \quad f_{2}(\zeta, \tau) \rightarrow 0, \quad \tau \rightarrow-\infty
$$

with no incident field at the left edge of the sample and with incident field at the left edge defined as follows:

$$
\begin{aligned}
& f_{1}(-10, \tau)=w \exp (i \theta), \\
& w=3.5 \exp \left[-\frac{1}{2}\left(\frac{\tau-3.0}{1.5}\right)^{2}\right], \\
& \theta=\arctan (\tanh [1.5(\tau-3.0)]) .
\end{aligned}
$$

In our case the spatial simulation domain was chosen as [-10,40]. Parameters $\Delta-\delta$ and $\mu$ were chosen

$$
\Delta-\delta=0, \quad \mu=1 .
$$

As one can see we gave the initial pulse the same configuration of phase in topological sense as in solitary wave solution. This point is important, because otherwise phase difference cannot relax to the symmetry of the stationary wave which is revealed in (1.64). As a result without right "topological charge" solution will be unstable.

For the second field boundary condition was as follows

$$
f_{2}(40, \tau)=0 .
$$

In the first experiment we injected a pulse relatively close to solitary wave solution. The results are shown in Figs. 1.2-1.4. The amplitude phase difference slightly differed as well as pulse shape which was Gaussian. On the first 


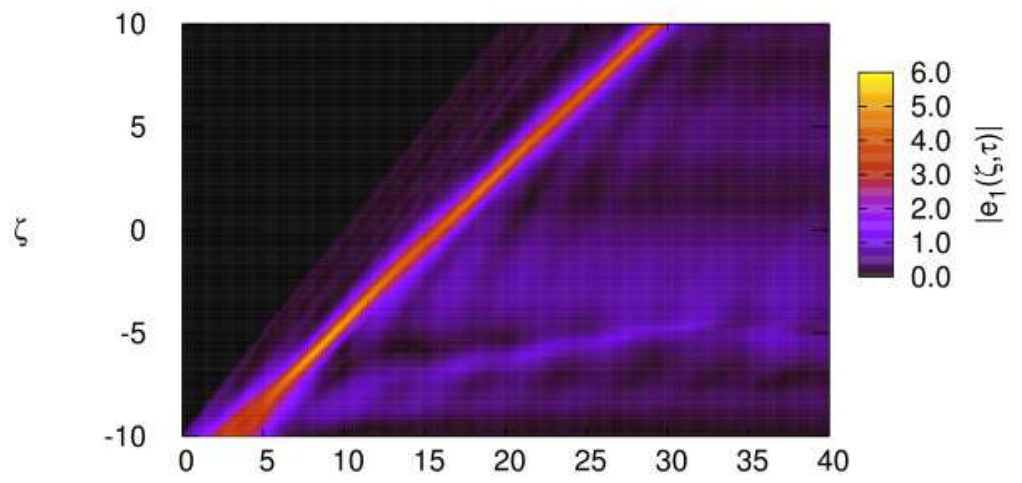

Fig. 1.2. Propagation of pulse. The first experiment. Mapping of the $\left|e_{1}(\zeta, \tau)\right|$ surface.

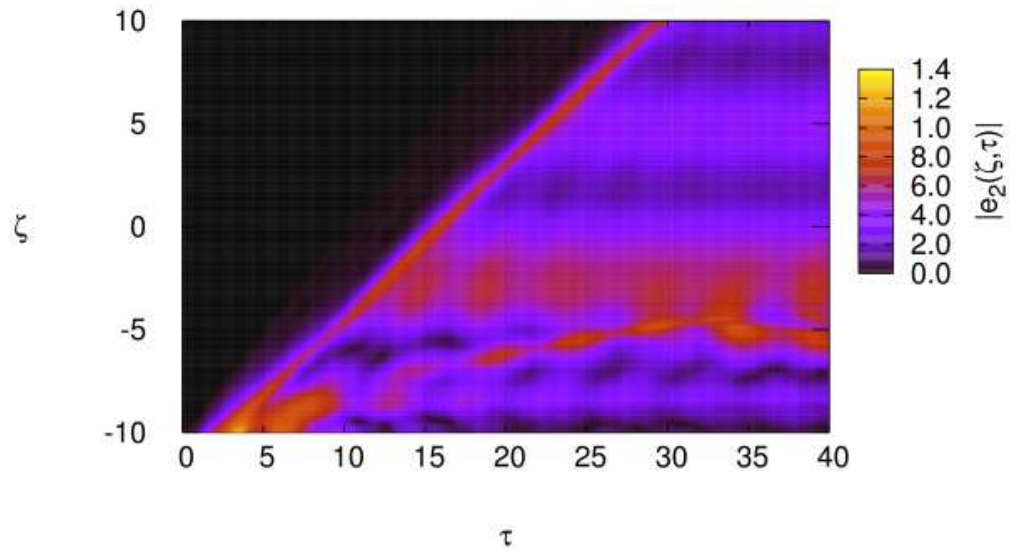

Fig. 1.3. Propagation of pulse. The first experiment. Mapping of the $\left|e_{2}(\zeta, \tau)\right|$ surface.

stage $(t \leq 7)$ of evolution we observed fast excess energy damping in radiation of quasi-linear waves in both directions and relaxation to solution roughly close to stationary one. Then we had some stage of pulse shape refinement $(7<t<30)$ with consequent propagation of the solution very close to $(1.63)$. One can compare refined pulse shape with stationary solution in Fig. 1.5.

During second experiment we used pulse of the lower amplitude

$$
\begin{aligned}
& f_{1}(-10, \tau)=w \exp (i \theta), \\
& w=2.0 \exp \left[-\frac{1}{2}\left(\frac{\tau-3.0}{1.5}\right)^{2}\right], \\
& \theta=\arctan (\tanh [1.5(\tau-3.0)]) .
\end{aligned}
$$




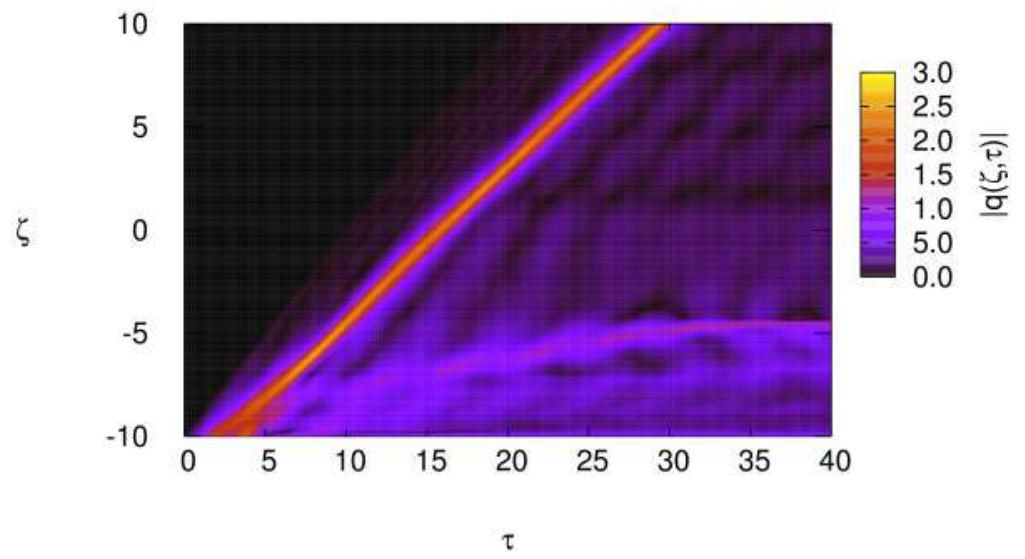

Fig. 1.4. Propagation of pulse. The first experiment. Mapping of the $|q(\zeta, \tau)|$ surface.

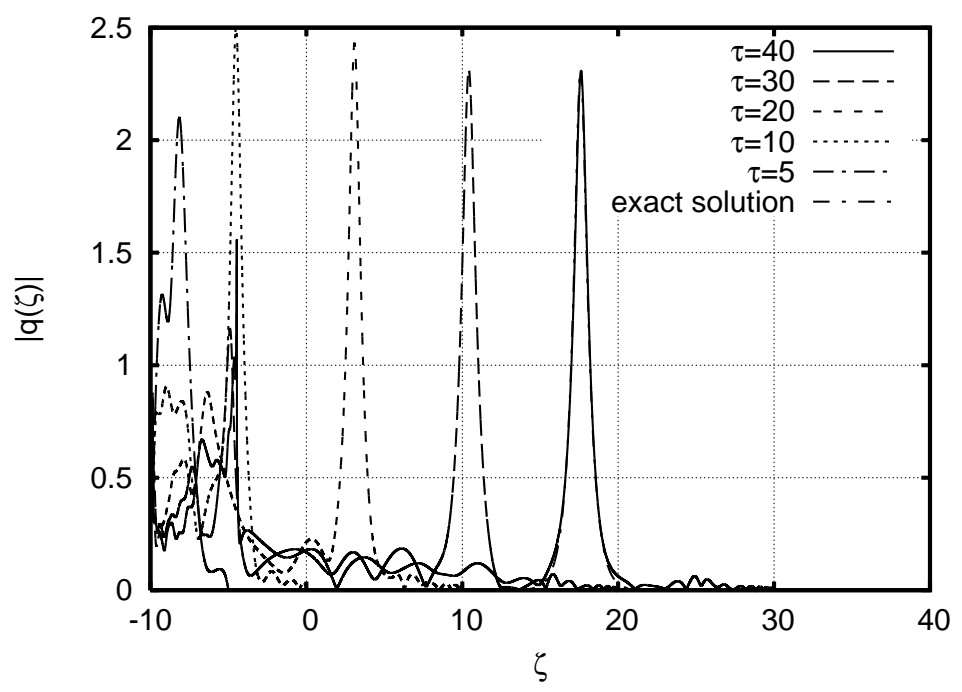

Fig. 1.5. The first experiment. Absolute value of polarization at different moments of time. Stationary pulse shape almost coincides with exact solution.

Results are represented in Figs. 1.6-1.8. Clearly observable oscillations of pulse magnitude can be associated with modes localized on pulse. This very instructive result could be a fruitful ground for further investigation and experiments. 


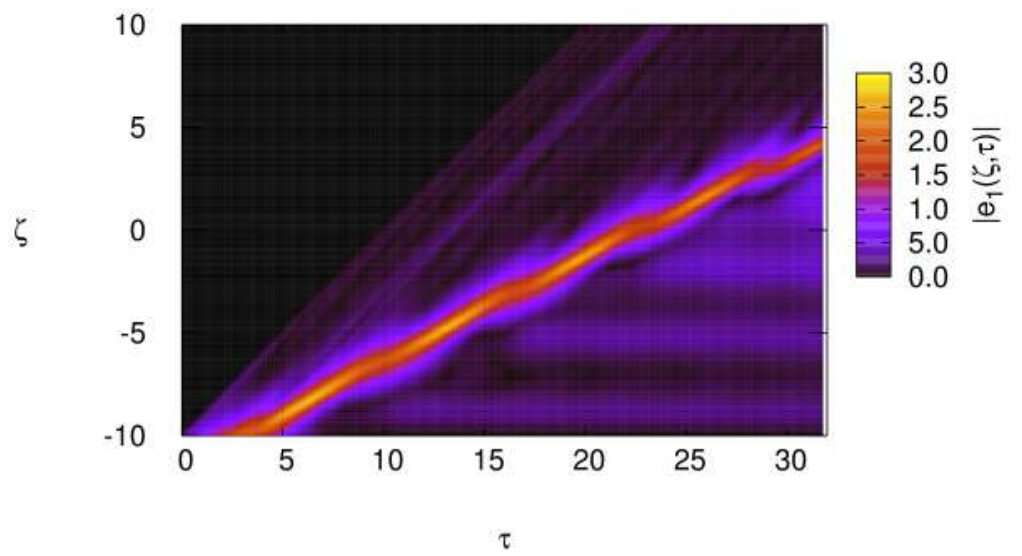

Fig. 1.6. Propagation of pulse. The second experiment. Mapping of the $\left|e_{1}(\zeta, \tau)\right|$ surface.

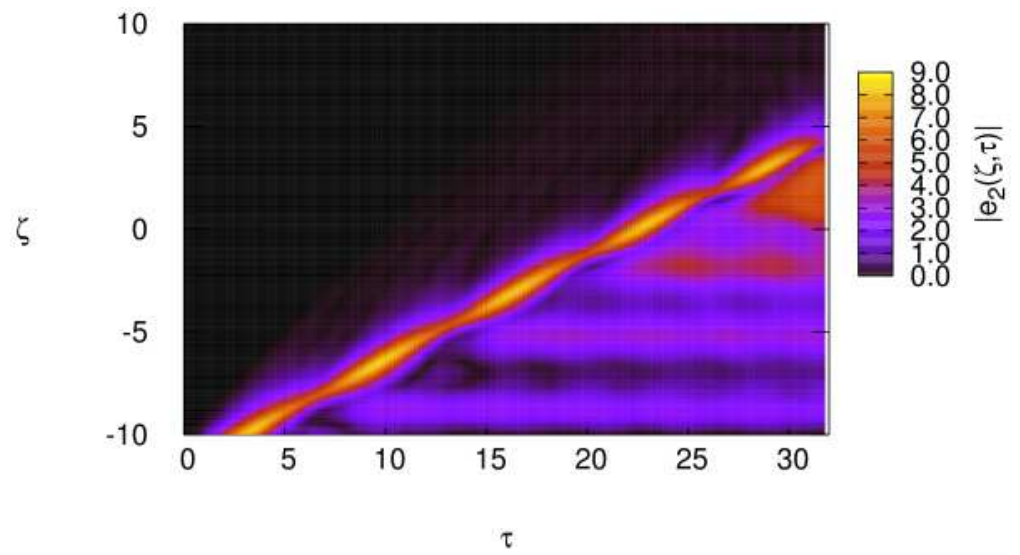

Fig. 1.7. Propagation of pulse. The second experiment. Mapping of the $\left|e_{2}(\zeta, \tau)\right|$ surface.

\subsection{Conclusion}

We derived equations describing optical pulse evolution in Bragg gratings with thin films containing active dopants. In particular we investigated case of thin films containing metallic nanoparticles. We showed that corresponding system have one parameter. This parameter contains information on Bragg and plasmonic frequencies and general pulse characteristics. These equations have solitary wave solutions describing bound state of two contraindicating waves with medium polarization. 


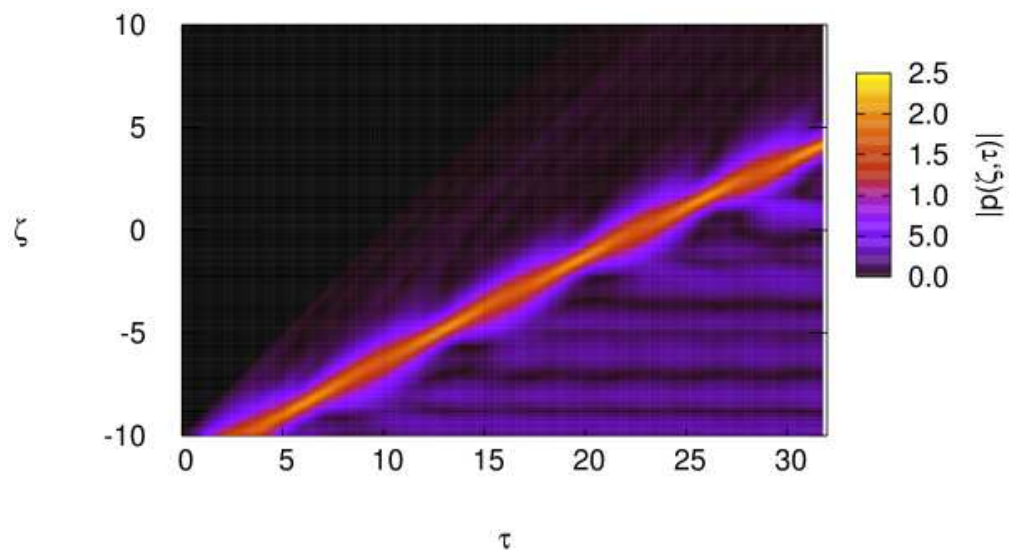

Fig. 1.8. Propagation of pulse. The second experiment. Mapping of the $|p(\zeta, \tau)|$ surface.

\section{Acknowledgments}

We would like to thank B.I. Mantsyzov, A. A. Zabolotskii, J-G. Caputo, M. G. Stepanov and R. Indik for enlightening discussions. AIM and KAO are grateful to the Laboratoire de Mathématiques, INSA de Rouen and the University of Arizona for hospitality and support. This work was partially supported by NSF (grant DMS-0509589), ARO-MURI award 50342-PH-MUR and State of Arizona (Proposition 301), RFBR grants 06-02-16406 and 0601-00665-a, INTAS grant 00-292, the Programme "Nonlinear dynamics and solitons" from the RAS Presidium and "Leading Scientific Schools of Russia" grant. KAO was supported by Russian President grant for young scientists MK-1055.2005.2.

\section{References}

1. B. I. Mantsyzov and R. N. Kuzmin, Sov. Phys. JETP 64, 37-44 (1986).

2. B. I. Mantsyzov and D. O. Gamzaev, Optics and spectroscopy 63, 1, 200-202 (1987).

3. T. I. Lakoba and B.I.Mantsyzov, Bull. Russian Acad. Sci., Phys. 56, 8, 12051208 (1992).

4. B. I. Mantsyzov, Phys.Rev. A51, 6, 4939-4943 (1995).

5. B. I. Mantsyzov and E. A. Silnikov, J. Opt. Soc. Amer. B, 19, 2203-2207 (2002).

6. A. Kozhekin and G. Kurizki, Phys. Rev. Lett. 74, 25, 5020-5023 (1995).

7. A. Kozhekin, G. Kurizki, and B. Malomed, Phys. Rev. Lett. 81, 17, 3647-3650 (1998).

8. T. Opatrny, B. A. Malomed, and G. Kurizki, Phys. Rev. E 60, 5, 6137-6149 (1999).

9. G. Kurizki, A. E. Kozhekin, T. Opatrny, and B. A. Malomed, Progr. Optics 42, 93-146 (E. Wolf, editor: North Holland, Amsterdam, 2001) 
10. J. Cheng, J. Zhou, Phys.Rev. E 66, 036606 (2002).

11. V.I. Rupasov, V.I. Yudson, Quantum electronics (in Russia) 9, 2179 (1982).

12. V.I. Rupasov, V.I. Yudson, Zh.E.T.Ph.(in Rusia) 93, 494 (1987).

13. P. Yeh, Optical Waves in Layered Media (Wiley, New York, 1988).

14. A.I. Maimistov, A.M. Basharov, Nonlinear Optical Waves (Dordrecht, Boston, London: Kluwer Academic Publishers, 1999).

15. L. Allen, J.H. Eberly, Optical resonance and two-level atoms, Wiley-Interscience, New York, 1975.

16. I.R. Gabitov, R.A. Indik, N.M. Litchinitser, A.I. Maimistov, V.M. Shalaev, J.E. Soneson, J. Opt. Soc. Am. B.23, 535-542 (2006)

17. A. I. Maimistov and V.V. Polikarpov, Quantum Electronics (in Russian) 36, 835 (2006).

18. A.A. Zabolotskii, private communication.

19. F. Calogero, A. Degasperis, in Solitons, R. K. Bullough, P. J. Caudray, eds. (Springer-Verlag, Berlin, 1980).

20. B. I. Mantsyzov, "Optical zoomeron as a result of beatings of the internal modes of a Bragg soliton", JETP letters, 82, 5, 253-258 (2005).

21. S. G. Rautian, "Nonlinear saturation spectroscopy of the degenerate electron gas in spherical metallic particles", JETP 85, 451-461 (1997).

22. V. P. Drachev, A. K. Buin, H. Nakotte, and V. M. Shalaev, "Size dependent $\chi^{3}$ for conduction electrons in Ag nanoparticles", Nano Lett. 4, 1535-1539 (2004).

23. F. Hache, D. Ricard, and C. Flytzanis, "Optical nonlinearities of small metal particles: surface-mediated resonance and quantum size effects", J. Opt. Soc. Am. B 3, 1647-1655 (1986).

24. C. M. De Sterke, J. E. Sipe, Prog. Opt. 33, 203 (1994).

25. R. Kashyap, Fiber Bragg Gratings (San Diego: Academic Press, 1999).

26. G. Agrawal, Y. S. Kivshar, Optical Solitons: From Fibers to Photonic Crystals (Amsterdam: Academic Press. 2003).

27. J. B. Pendry, A. J. Holden, D. J. Robbins, W. J. Stewart, IEEE Trans. Microwave Theory Techniques 47, 2075-2084 (1999).

28. D. R. Smith, S. Schultz, P. Markos, C. M. Soukoulis, Phys. Rev. B 65, 195104 (2002).

29. N. Katsarakis, T. Koschny, M. Kafesaki, E. N. Economou, C. M. Soukoulis, Appl. Phys. Lett. 84, 2943-2945 (2004).

30. P. Markos, C. M. Soukoulis, Phys. Rev. E 65, 036622 (2002).

31. J. F. Woodley, M. S. Wheeler, M. Mojahedi, Phys. Rev. E 71, 066605 (2005).

32. N. M. Lichinitser, I. R. Gabitov, A. I. Maimistov, V. M. Shalaev, "Effect of an optical negative index thin film on optical bistability", arXiv. physics/0607177.

33. A. A. Zharov, I. V. Shadrivov, Yu. S. Kivshar, Phys. Rev. Lett. 91, 037401 (2003).

34. A. I. Maimistov, "On Coherent Optical Pulse Propagation in One-Dimensional Bragg Grating", arXiv. nlin.PS/0607012

35. R.W. Ziolkowskii, E. Heyman. Phys. Rev. B 64056625 (2001).

36. R.W. Ziolkowski, Optics Express 11, 21167, 662 - 681 (2003). 